\title{
Concepts, intelligence, creativity and coping
}

Natalia Eduardovna Volkova

Institute of Psychology of the Russian Academy of Sciences

\section{Abstract}

The relation of conceptual structures, intelligence, creativity and coping has not yet been the subject of a psychological research. Our study aimed to reveal interrelations between the features of the organization of the concept with intelligence creativity and coping. A complex design was used in our research including assessment of (a) IQ (SPM); (b) creativity (TTCT); (c) coping strategies; (c) the semantic differential technique (SD) (stimulus word "potential").

Participants of the experiment were 408 students aged 18-2 years (mean $19,32 \pm 1,48$ ) among them $79,2 \%$ of girls.

We processed the data by mathematical and statistical approach: primary data that were collected from eligible respondents were subjected to be analyzed using linear regression. The findings revealed that the concept "potential" has considerable number of reliable interrelations both with indicators of the general abilities (IQ, creativity) and with coping strategies.

It was shown that the maximum saturation of the concept with sensory-emotional features can lead to a decrease in the productivity of intellectual activity. It should be noted that the role of the considered indicators of concepts in efficiency of intellectual activity is slightly lower, than in creative activity. It was found that between the variable measures of differentiated participation of sensory-emotional features of the concept and indicators of coping strategies there is quite close, but at the same time, multidirectional relationship.

Thus, we may conclude that conceptual structures may act as the intermediary in relation to intelligence, creativity and coping strategies. The finding of our research may be usefu for solving the problem of human mental resources increasing through the development of conceptual thinking.

This research was funded by Russian Foundation of Basic Research, 2018-2020, Research grant 19-013-00294 A.

\section{Introduction}

One of the key directions in the study of human ature is the search for an answer to the question of the mechanisms of reasonableness as the basis or effective cognitive activity and constructive social life of people.

Many researchers have recognized the special ole of concepts in the structure of intelligence, considering the ability to conceptual reflection as the highest stage of intellectual development, providing the possibility of conscious (arbitrary) regulation of activity

\section{Research Question} at impact conceptual structures have on Frydenberg in T.A. Krjukova's variations of indicators of conceptual abilities, adaptation.

cognitive abilities creative abilities and on coping

strategies on people in young adulthood?

\section{Purpose of the study}

The purpose of this empirical research is to identify the impact of indicators of conceptual structures (sensory-emotional signs) on variations of conceptual (voluntary categorical, nvoluntary categorical and generative) abilities, as well as on variations of cognitive abilities (level of analytical intelligence) and on variation of creative abilities (verbal and non-verba creativity), and on variations of indicators coping strategies in adolescence.

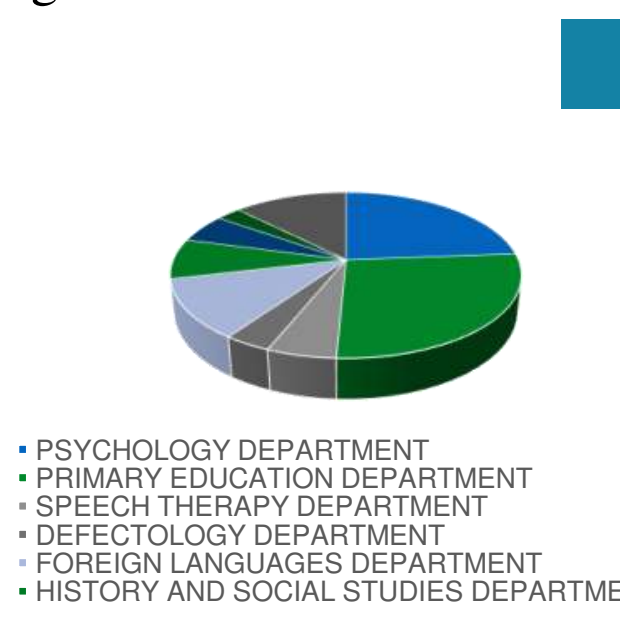

\section{Methodology}

A complex design was used in our research including:

1) modification of "Integral Conceptual Structures" technique,

2) conceptual abilities technique ("Conceptual thinking" technique),

3) creativity (E.P. Torrance technique (both TTCT-Figural and TTCT-Verbal))

4) Raven's intelligence test (SPM )

5) ACS- Adolescent Coping Scale E

(n)

Statistical data processing

Statistical data processing was carried out based on the IBM SPSS software package. Statistics 22.0. It included descriptive analysis (mean, standard deviation) and analysis of variance (Linear Regression Modelling (Stepwise method)).

\section{Participants} Geographical profile of respondents

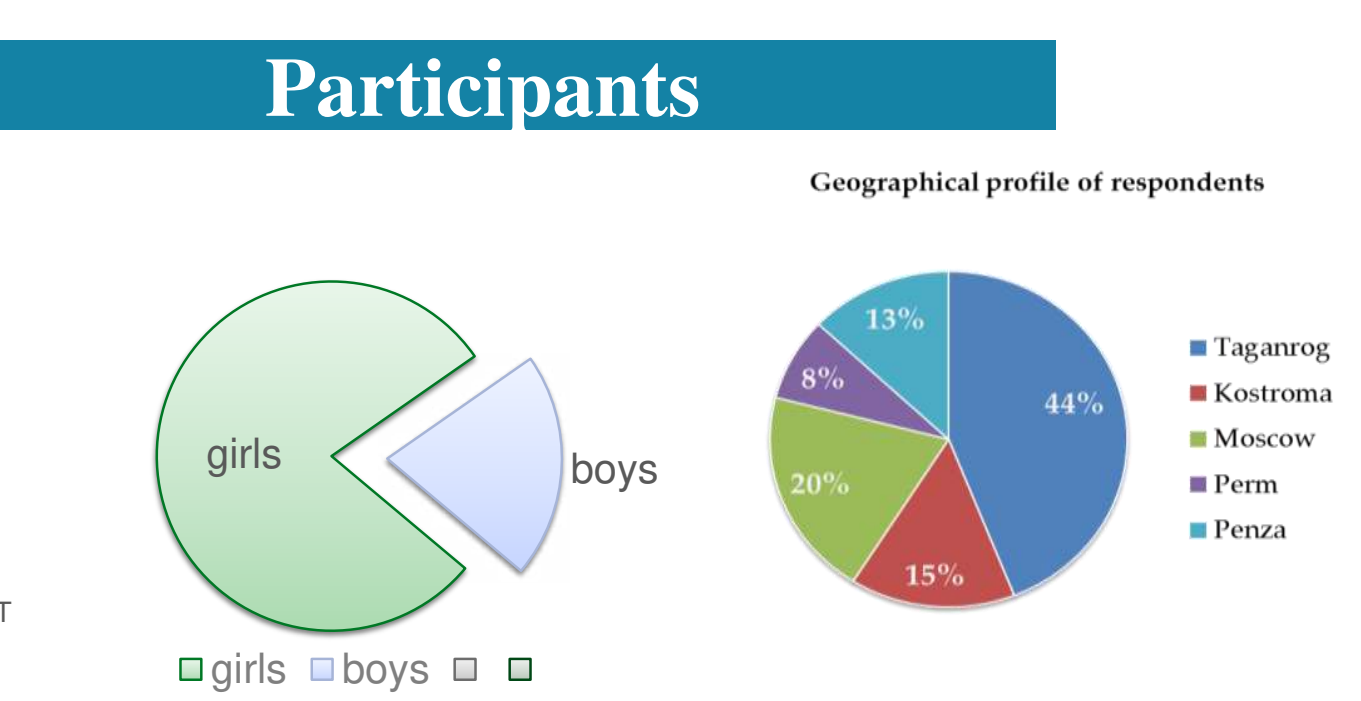

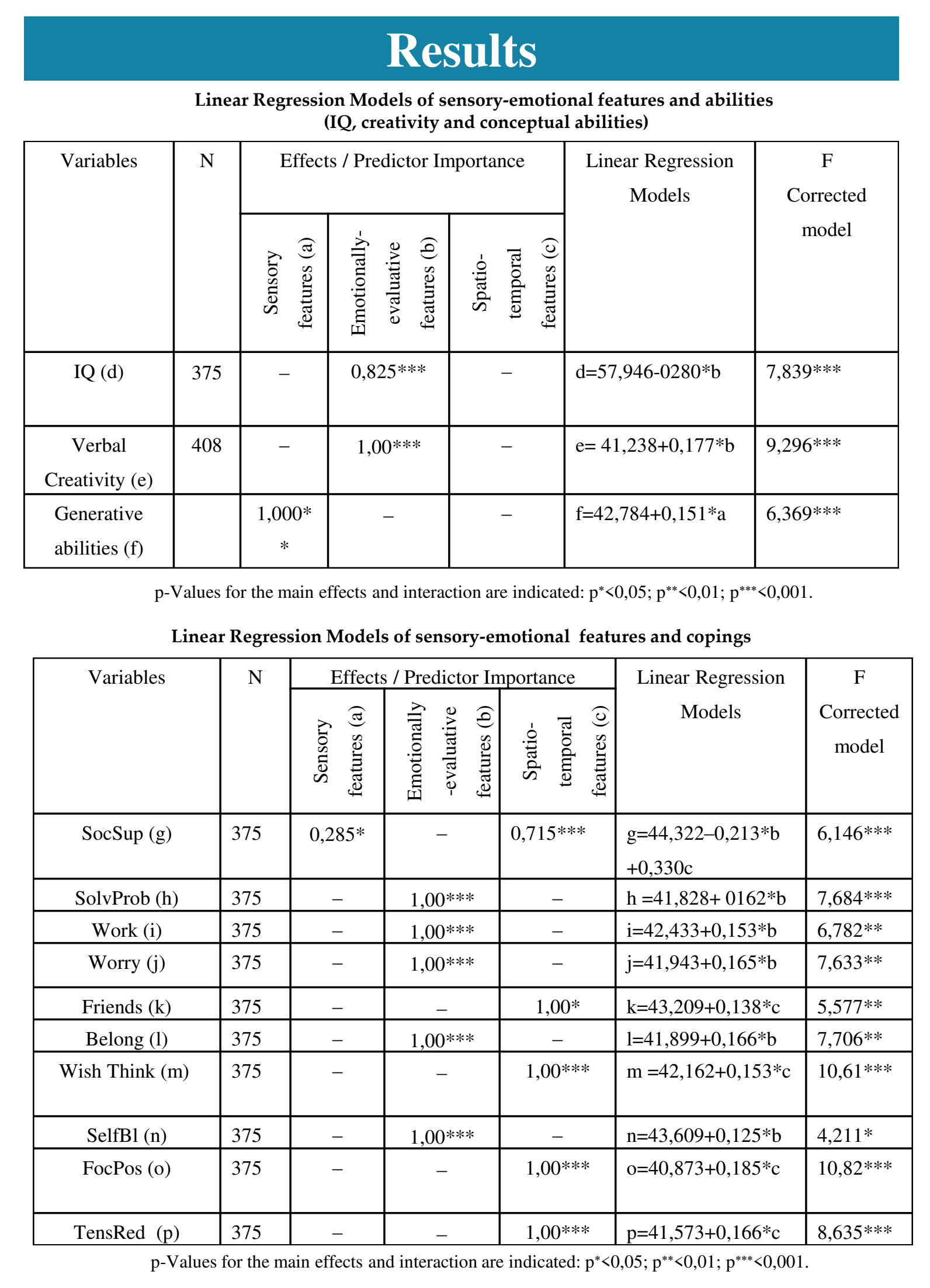

Conclusion

The data obtained show that sensory-emotional experience in the concepts is different and can be linked both with personal traits and the peculiarities of the professional education.

\section{Acknowledgements}

Institute of Psychology of the Russian Academy of Sciences Correspondence: nats29@ mail.ru; Tel.: +7916-032-48-06 